teienwettbewerb findet der Leser nicht. Bedauerlich ist dies besonders, wenn es um Reformoptionen geht. Die Reformbedürftigkeit der Finanzverfassung wird nur aus der Verschuldung abgeleitet. Wirkliche oder vermeintliche Verwerfungen werden zwar behauptet, aber kaum belegt oder begründet (S. 212 ff.). Dies gilt auch für die „Vorgaben für künftige Reformen der Finanzordnung". Dabei unterbleibt leider die Befassung mit zentralen Fragen der bis 2019 anstehenden Reform des Finanzausgleichs, etwa die Berücksichtigung des demografischen Wandels oder die ungleichmäßig streuenden Belastungen der Länder durch die Bundespolitik. Stattdessen wird zum wiederholten Male die Debatte über eine Länderneugliederung geführt. So kann Buschers Arbeit nicht die Standardwerke ersetzen.

Der Titel der von Axel Heise vorgelegten Untersuchung führt in die Irre: Angekündigt wird eine vergleichende Analyse der bundesstaatlichen Finanzbeziehungen, geliefert wird eine tour d'horizon verschiedener Aspekte bundesstaatlicher Ordnungen, die alle irgendwie mit Finanzen zusammenhängen. Auf 18 Seiten wird das Thema Föderalismus und Finanzordnung abgehandelt; auf 14 Seiten erfährt der Leser etwas über die Entwicklungsphasen von Föderalismus und Finanzbeziehungen; der föderalen Struktur und den Finanzbeziehungen werden immerhin 27 Seiten gewidmet, wobei unter "Struktur" so disparate Aspekte wie föderale Beziehungen, Regierungssystem, Parteiensystem, Politische Kultur, Steuererhebung und Steuereinnahmen, Solidarität im Bundesstaat und „die Ausgabenseite“ subsumiert werden. Schließlich geht Heise auf knapp 20 Seiten anhand von sechs Beispielen der Frage nach, warum es in den drei genannten Staaten Aufsteiger- und Absteigerregionen gibt. Im Ergebnis stellt er fest, dass die Frage nach den Gründen für Erfolg oder Misserfolg einer Region nicht eindeutig beantwortet werden kann.

Auffällig, teilweise unerfreulich ist, dass der Verfasser Analyse und Wertung nicht hinreichend trennt. Gleich zu Beginn der Arbeit (S. 11) werden die gängigen kritischen Schlagworte aneinandergereiht, der „Spiegel“ zitiert und diese Fundstücke als Grund für die beiden Föderalismuskommissionen ausgegeben. Flapsige Formulierungen, in denen den Ministerpräsidenten „Angst vor der eigenen Courage“ (S. 12) vorgehalten wird, stärken nicht die wissenschaftliche Solidität. Kontroverse Meinungen und Positionen im wissenschaftlichen Diskurs werden arbiträr verwandt und als gesichert dargestellt. Insgesamt bleibt der Erkenntnisgewinn gering. Eigentlich schade, denn die im zweiten Teil aufgeworfene, aber unzureichend behandelte Frage nach den Ursachen von Erfolg und Misserfolg von Regionen ist wichtig.

Wolfgang Renzsch

Föderalismus in Deutschland und der Welt: gelungener Überblick

Sturm, Roland: Föderalismus. Eine Einführung, 2. Auflage, Nomos Verlagsgesellschaft, BadenBaden 2010, 242 Seiten, $€ 19,90$.

Obwohl der Föderalismus nicht nur in Deutschland als Strukturprinzip der politischen Ordnung eine lange Tradition hat, sondern auch weitere Staaten Europas Reformen in Richtung eines föderalen, jedenfalls aber dezentralen Systems unternehmen (Italien, Großbritannien) beziehungsweise unternommen haben (Belgien, Spanien), sind Überblickswer- 
ke über die theoretischen Grundlagen und die realen Ausformungen des Föderalismus noch immer Mangelware. Bereits 2005 hatte Roland Sturm gemeinsam mit Petra ZimmermannSteinhart eine Einführung in den deutschen Föderalismus vorgelegt, der 2010 eine zweite Auflage erfahren hat (bei der Petra Zimmermann-Steinhart allerdings nicht mehr als KoAutorin aufgeführt wird, wenngleich ihre Mitarbeit im Untertitel erwähnt wird).

Sturm beginnt mit einer Erläuterung der theoretischen und systematischen Grundlagen, insbesondere zentraler Begriffe wie „Bundesstaat“ und „Subsidiarität“, die für das Verständnis nicht nur des deutschen Föderalismus unverzichtbar sind. Auch die normative und empirische Herangehensweise der Föderalismusforschung wird kurz skizziert. In diesem Kapitel wird deutlich, dass es Sturm vorwiegend um die Darstellung des deutschen Falles geht, denn aus ihm bezieht er die meisten Beispiele, die der Illustration der verschiedenen Aspekte des Föderalismus dienen.

Diese Orientierung setzt sich im Folgenden zwar fort; zugleich werden aber immer wieder Bezüge zu anderen Bundesstaaten (wie der Schweiz, Kanada oder den USA) hergestellt. Dies gilt insbesondere für das zweite und dritte Kapitel, in denen es um die institutionellen Merkmale des Föderalismus und die Finanzordnung geht. Spätestens im Abschnitt über die Institutionen wird auch klar, warum der erst vor gut fünf Jahren erschienene Band einer Aktualisierung bedurfte: Sie wurde erforderlich durch die schon im Unterabschnitt „Kompetenzverteilung und Mehrebenenpolitik" sowie später noch in einem eigenen Kapitel (5) ausführlich diskutierten Hintergründe, Ergebnisse und „offenen Flanken“ der Föderalismusreformen 2006 und 2009 in Deutschland. Im Mittelpunkt dieses Kapitels steht ansonsten die wiederum sehr viel stärker international vergleichend angelegte Diskussion der Rolle Zweiter Kammern im Bundesstaat. Hier nimmt Sturm besonders für Deutschland die jüngst von Karlheinz Niclauß nachgewiesene und nur zu unterstreichende These auf, der Bundesrat sei bereits vom Parlamentarischen Rat nicht als Widerlager „zur“, sondern „in“ der Parteipolitik konstruiert worden. Vor allem aber werde, wie Sturm zu Recht betont, immer noch der territoriale Aspekt der Entscheidungsfindung im Bundesrat zu selten beachtet (S. 67 f.). Wie allerdings die Vereinbarung von Abstimmungsverhalten in Landeskoalitionen nach Öffnung des Abstimmungsverfahrens im Bundesrat für eine Art von „Stimmensplitting" genau umzusetzen wäre, lässt Sturm offen (S. 71). Das Kapitel wird mit einer kurzen Betrachtung der Rolle der Landesparlamente fortgeführt, für deren Relevanz als Legitimationsinstanz er eine ebenfalls überzeugende Lanze bricht. Es endet mit einer Darstellung der Probleme, die den Kommunen aus der spezifisch deutschen Ausprägung des Föderalismus erwachsen.

Letzteres führt fast schon logisch zum Thema des nächsten Kapitels: die Finanzverfassung. Bekanntlich - man denke nur an die immer wieder aufflammenden Diskussionen über Sinn und Unsinn des Länderfinanzausgleichs - ist es diese, die über die tatsächlichen Möglichkeiten des Bundes und vor allem der Länder zu eigenständiger Politikformulierung entscheidet. Hier könnte man den Klassiker „no taxation without representation“ fast in ein „keine Aufgabenübertragung ohne Mittelzuweisung“ umformulieren. Der Skepsis, die Sturm schließlich gegenüber der neu eingeführten Schuldenbremse auf Bundes- und Landesebene durchklingen lässt, ist bei aller Einsicht in die Notwendigkeit der Rückführung von Schulden (die damit ja gar nicht gewährleistet wird) im Grundsatz zuzustimmen (S. 122 - 131).

Auch stärker auf Deutschland rekurriert Sturm im Kapitel über die „Politische Willensbildung“, in dem er zum einen die Möglichkeiten direkter Demokratie, zum anderen jene der „klassischen“ parlamentarisch-repräsentativen Demokratie im Zeichen des modernen 
Parteienstaats darstellt. Im fünften Kapitel stehen, wie bereits erwähnt, die beiden abgeschlossenen Föderalismusreformen von 2006 und 2009 im Mittelpunkt, ergänzt um prospektiv formulierte Überlegungen zu einer „dritten Föderalismusreform“ (S. 208), bei denen vor allem die Frage nach den Folgen der beiden ersten Reformen angerissen wird.

Das Buch endet mit einem kurzen Blick in vier Nachbarstaaten Deutschlands (Großbritannien, Frankreich, Polen, Tschechische Republik), die sich von stark zentralisierten Einheitsstaaten auf den Weg zu einer größeren Dezentralisierung, möglicherweise auch Föderalisierung ihrer politischen Systeme begeben haben, wobei letzteres aber nach Ansicht von Sturm noch nicht der Fall zu sein scheint.

Das Buch ist in der Reihe „Studienkurs Politikwissenschaft" des Nomos-Verlages erschienen und wird dem Anspruch der Herausgeber dieser Reihe, einen „schnellen und einführenden Einstieg in Grundfragen und -themen der Politikwissenschaft" (Homepage des Nomos-Verlages) zu eröffnen, gerecht. Insbesondere für Studierende sind die bibliographischen Hinweise und „Kontrollfragen“ am Ende der Kapitel hilfreich. Aber auch für den interessierten Fachwissenschaftler ist der Band mit seinem aktuellen Überblick über den Stand der Föderalismusforschung lohnend.

Sven Leunig

\section{Parteiendemokratie in Deutschland: Absage an den Abgesang}

Liedhegener, Antonius und Torsten Oppelland (Hrsg.): Parteiendemokratie in der Bewährung. Festschrift für Karl Schmitt, Nomos Verlagsgesellschaft, Baden-Baden 2009, 574 Seiten, € 89,-.

Die Parteien gehören in allen modernen Demokratien zu den wichtigsten Trägern der politischen Ordnung. Doch die Zeichen mehren sich, dass das Modell der Parteiendemokratie, im 20. Jahrhundert zur erfolgreichsten Form repräsentativer Demokratie aufgestiegen, zunehmend unter Anpassungsdruck gerät. Einerseits schwinden die Handlungsspielräume der Nationalstaaten durch Globalisierung und europäische Integration; andererseits erwartet die Bevölkerung, durch neue Formen politischer Beteiligung eingebunden zu werden, während gleichzeitig das Vertrauen in Parteien und politische Eliten, auf dem die Legitimität der repräsentativen Demokratie beruht, nachhaltig in Mitleidenschaft gezogen ist. Das bisher erfolgreiche Modell, so die Herausgeber, scheint heute „in eine erneute Phase der Bewährung eingetreten“; junge wie etablierte Parteiendemokratien müssen ihre Stärke unter deutlich veränderten Bedingungen beweisen (S. 12).

Der Sammelband zieht eine vergleichende Bilanz der historisch wie aktuell beobachtbaren Erfahrungen mit der Parteiendemokratie - im Sinne Karl Mannheims als Gleichzeitigkeit des entwicklungsgeschichtlich Ungleichzeitigen. Er spannt dazu einen weiten Bogen, der von der historischen Entwicklung des deutschen Parteienstaats in der Weimarer Republik bis heute (1. Kapitel), über die Bedeutung der Parteien im Institutionengefüge der Bundesrepublik (2.), die Entwicklung des deutschen Parteiensystems und die Diskussion um die „Krise der Parteien“ (3.), die in Thüringen beziehungsweise in den jungen Bundesländern entstandene Parteiendemokratie (4.), die Entwicklung der Parteiendemokratie in den westlichen (5.) und den nachholenden Demokratien (6.) bis zu einem Ausblick auf die wachsende internationale Ausrichtung der Parteien im 7. Kapitel reicht. Mit dieser Festle- 